\title{
Las bibliotecas rurales en los medios de comunicación de la Región de Extremadura: un estudio de caso
}

\author{
Raquel Cebrián-Solís \\ Cristina Faba-Pérez
}

Recibido: 3 de noviembre de 2015

DOI: http://dx.doi.org/rev_CDMU.2016.v27.51794

Aceptado: 28 de enero de 2016

\section{Resumen}

El objetivo principal de este trabajo es, a través de las noticias publicadas en diversos medios de comunicación de la Región de Extremadura sobre bibliotecas rurales a lo largo de los últimos años, comprobar el impacto y la visibilidad de las bibliotecas en los medios. Рara ello, se recopilan un total de 1196 noticias que, tras ser analizadas detalladamente, se reducen a 300 sobre las que versan los resultados del estudio. Esta disminución de noticias finales demuestra que el titular o los primeros párrafos no expresan exactamente el contenido de la noticia siendo necesario un análisis cualitativo de su contenido. Se concluye que: las noticias sobre bibliotecas rurales han decrecido en el periodo analizado (2007, 2008, 2012, 2013), siendo 2007 el año de mayor auge, si bien se ha observado un repunte en 2013 con respecto a 2012; las noticias sobre bibliotecas rurales de ámbito provincial (Badajoz y Cáceres) obtienen mayor porcentaje que las regionales y nacionales (sin representación); y el diario HOY es, con diferencia, el medio que acumula el mayor porcentaje de noticias sobre bibliotecas rurales.

\section{Palabras clave}

Medios de publicación, bibliotecas rurales, impacto, visibilidad, análisis, Extremadura.

\section{Rural libraries in the mass media in Extremadura : a case study}

\section{Abstract}

The primary objective of this work was to study the evolution of news items about rural libraries published in the different communications media of the Region of Extremadura (Spain) in recent years, to see whether the country's economic situation also affected the number of such news items and their visibility in the media. For this, a total of 1196 news articles were collected. After their analysis in detail, these were reduced to the 300 which the study's results address. This shows that the title or the first few paragraphs of the news does not exactly express its content. The conclusion reached is that: Although the numbers of those items decreased in the period analysed (from 2007 and 2008 to 2012 and 2013), there was an upswing in 2013 compared with 2012; News on rural libraries at provincial level (Badajoz and Cáceres) obtain higher percentage than 
the regional and national news; The Newspaper HOY is the medium that accumulates the highest percentage of rural libraries news.

\section{Keywords}

Print media, rural libraries, impact, visibility, analysis, Region of Extremadura.

\section{Introducción}

Henry Ward Beecher ${ }^{1}$ (1813-1887), dijo: "Una biblioteca no es un lujo, sino una de las necesidades de la vida". Reflexionando sobre esta cita no se entiende cómo en estos tiempos actuales no se valora como se debiera a las bibliotecas más pequeñas, las bibliotecas rurales, es decir, las bibliotecas de los pueblos pequeños, puesto que, tal y como señalan Hidalgo Mateos y sus colaboradores (2012: p. 7) "Las bibliotecas rurales (...) se han convertido en uno de los pilares del patrimonio cultural colectivo". Si bien es cierto que tiempo atrás, después de proclamarse la II República en España, la cultura se entiende como un bien común para todos, especialmente en las zonas rurales, y las pequeñas bibliotecas se convierten en un elemento fundamental para permitir que el libro llegue a los rincones más apartados de nuestro país (Boza Puerta y Sánchez Herrador, 2004: pp. 41-47), también lo es que las bibliotecas de localidades más pequeñas (rurales) no han sido motivo de numerosos estudios y, menos aún, en zonas como Extremadura.

Por ello, el objetivo principal que se plantea esta investigación es estudiar la cantidad de noticias publicadas en diversos medios de comunicación de la Región de Extremadura sobre bibliotecas rurales a lo largo de los últimos años, con el fin de comprobar el impacto y la visibilidad de este tipo de bibliotecas en los medios. Se ha querido subrayar la importancia de las bibliotecas rurales (bibliotecas públicas que suministran sus servicios solo a las poblaciones en Extremadura entre 1000 y 5000 habitantes) ${ }^{2}$ por dos motivos: en primer lugar, porque en términos demográficos Extremadura es la Región de España con mayor porcentaje de habitantes residiendo en municipios rurales; y en segundo lugar, porque, si bien las investigaciones sobre bibliotecas rurales en otros países, e incluso en España, son considerables, sin embargo, las investigaciones sobre 1Información y biografía sobre Henry Ward Beecher (disponible en: http://www.qotd.org/quotes/Henry.Ward.Beecher). 2Ley 6/1997, de 29 de mayo, de Bibliotecas de Extremadura (BOE. núm. 188, de 7 de agosto de 1997). 
ellas en Extremadura son muy escasas.

Con respecto a la ruralidad de Extremadura, para García Gómez y Díaz Grau (2001: p. 145), "se considera medio rural aquellas zonas caracterizadas por problemas de accesibilidad, infraestructuras deficientes y un desarrollo pésimo de servicios públicos. Todo ello acompañado de escasos niveles de ingresos, escasez de ofertas de empleo o emigración de la población más joven hacia áreas urbanas". Si se analiza la clasificación de las Comunidades Autónomas en España atendiendo a su ruralidad ${ }^{3}$, los porcentajes de territorio y de municipios rurales superan el $90 \%$ en las Comunidades de Castilla y León, Aragón, Extremadura, Castilla-La Mancha, la Rioja y Navarra. En términos demográficos, es Extremadura la Comunidad con un mayor porcentaje residiendo en municipios rurales, nada menos que el 88,3 , seguida de Castilla-La Mancha $(77,4 \%)$ y Castilla y León (44,9\%). Es decir, Extremadura es predominantemente rural.

Con respecto al segundo motivo, es decir, a las investigaciones sobre bibliotecas rurales, a escala internacional, hay trabajos sobre bibliotecas rurales en Australia (Haines y Calvert, 2009), US (Mehra et al., 2010), Finlandia (Wigell-Ryynänen, 2009), Francia (Germanaud y Rapaport, 1988), Israel (Hoffman-Pfeffer, 1987) o China (Yuan, 2011). En el caso de España, también se han realizado investigaciones sobre bibliotecas rurales de diferentes zonas geográficas como Andalucía (Busutil Rivera, 2011), Álava (Gómez Santamaría, 2009), Asturias (Busto Fidalgo, 2009), Barcelona (Cano Vers, 2009), CastillaLa Mancha (Lorenzo Jiménez, 2009), Madrid (Cid Ruiz y Fraile de la Hoz, 2009) o Valencia (Moliner Ruiz, 2010). Sin embargo, en la Región de Extremadura se ha observado una ausencia de publicaciones en materia de bibliotecas rurales. El único estudio realizado recientemente es el coordinado por Hidalgo Mateos en el año 2012 en el que se analizan 182 bibliotecas rurales extremeñas (Hidalgo Mateos et al., 2012). La imagen de las bibliotecas rurales en la prensa española tampoco ha sido demasiado analizada y, por supuesto, actualizada, muestra de ello es un trabajo realizado en 1997 por Gómez Gómez, Gómez Hernández y Lluch Baixauli (1997) sobre La imagen de las Bibliotecas Municipales en la prensa regional de Murcia, en el que desglosa el número de noticias por municipios.

3Plan Estratégico Nacional de Desarrollo Rural 2007-2013 (disponible en: http://www.magrama.gob.es/es/desarrollorural/temas/programas-ue/PENv3_23-05-12_tcm7-9908.pdf). 
Antes de continuar también es preciso matizar que, aún en la actualidad, no existe una definición precisa y generalmente aceptada de las bibliotecas rurales o bibliotecas públicas del medio rural, aunque Ford (2002: p. 192), señala que "...una biblioteca pública bien empleada puede realizar una notable aportación a la vitalidad de las áreas urbanas y convertirse en un importante centro social y educativo, además de punto de encuentro, sobre todo en áreas rurales dispersas". García Gómez (2004: p. 9) define a la biblioteca en el mundo rural como: "...es un servicio completo donde se le da un especial énfasis a la creación y difusión de información local y comarcal, donde se desarrollen servicios perfilados a las características y necesidades de cada núcleo rural en colaboración con otras entidades, para poder responder en la medida de lo posible a las particularidades propias de la ciudadanía, lo que favorecerá y potenciará la imagen del servicio bibliotecario prestado".

En el medio rural hay mucha necesidad de información, por lo que es preciso acercar el acceso a la cultura a sus gentes, a la vez que motivar su desarrollo personal y social. Llano Caelles (1997: p. 13), en su estudio sobre las bibliotecas y el medio rural, considera que: "(...) la biblioteca pública, en cuanto al servicio público de calidad que se presta a personas de manera individualizada, puede cobrar una relevancia especial en el proceso de desarrollo y diversificación socio-económica de las comarcas rurales". Evidentemente, tal y como señalan las Directrices IFLA/UNESCO para el desarrollo del servicio de bibliotecas públicas (2001: p. 25), "los servicios biblioteconómicos han de estar adaptados a las necesidades de las distintas comunidades rurales y urbanas". Por ello, en el caso concreto de las bibliotecas de las zonas rurales, según García Gómez y Díaz Grau (2001: p. 151), éstas deben ofrecer los siguientes servicios:

- Servicio de lectura.

- Servicio de dinamización y extensión cultural.

- Servicio apoyo promoción de empleo.

- Servicio de nuevas tecnologías.

- Servicio de formación.

- Servicio de información.

- Servicio apoyo centros educativos.

Comalat Navarra y Reyes Camps en 2001 (pp. 26-27), apuntan como servicios, tanto el 
espacio cultural y de encuentro, como el de construcción de la identidad local. En relación con el espacio cultural y de encuentro "... proponen ofrecer una vida cultural más rica a todos los habitantes de estas poblaciones (...) actividades, que lógicamente, tienen que ir muy ligadas a las necesidades y a los intereses de cada municipio, no sólo serán una manera de acercar a usuarios potenciales a otros servicios de la biblioteca, sino que su pertenencia tiene que asegurar que sea embrión o motor para el desarrollo cultural del municipio". El servicio del espacio de encuentro se elaboró debido "...al hecho de ser el trabajo de campo sumamente solitario, así como la existencia de población diseminada, se le ha añadido el traslado por motivos laborales de parte de la población. Estas personas necesitan tener un espacio de encuentro para relacionarse. Esta función es la que han tenido históricamente los casinos, a pesar de que generalmente únicamente la han disfrutado los hombres." Por otro lado, en lo referente a la construcción de la identidad local "...este servicio de la biblioteca pública ha sido, en nuestros ámbitos geográficos, poco trabajado por falta de recursos y por prioridad de oferta de otros servicios; en estos momentos, sin embargo, es preciso incidir y prever en él de una intervención futura importante."

\section{Datos y Metodología}

Actualmente, la información es uno de los bienes más preciados para que se produzca un crecimiento en la economía, en la sociedad y en la cultura de una región, siendo las bibliotecas el germen que permite al ciudadano introducirse en el mundo de la globalización informativa (González-Mateos y Faba-Pérez, 2013) y, más aún, en zonas geográficas aisladas como es el caso de Extremadura. Pero actualmente también, se vive en un momento donde los medios económicos son más escasos por motivo de la crisis, por lo que hay que intentar, con menos o los mismos recursos, conseguir excelentes resultados en las bibliotecas de ámbito rural. Ello solo se logrará realizando trabajos eficientes y eficaces que demuestren la necesidad de este tipo de bibliotecas en zonas apartadas de la geografía española y, en especial, extremeña. Por esto, en el presente artículo se profundiza en el estudio de las bibliotecas rurales desde diversos puntos de vista con el fin de aumentar la visibilidad de las mismas. Es imprescindible la comunicación de la biblioteca rural con la comunidad y la necesidad de su presencia en 
los medios para ser referencial y estar al servicio de la comunidad cumpliendo sus fines, por ello, como se muestra a continuación, se analizan con detalle las noticias sobre bibliotecas rurales aparecidas en varios medios de publicación extremeños.

Para obtener el conjunto de noticias sobre bibliotecas rurales, o bibliotecas públicas del medio rural, se seleccionan tres medios de publicación que representan ampliamente a la Región de Extremadura por los motivos siguientes:

El Diario Oficial de Extremadura (DOE) (http://doe.juntaex.es/), por ser el Boletín Oficial de la Región, es decir, el medio para dar publicidad a los documentos que, de acuerdo con el ordenamiento jurídico y en virtud de los principios constitucionales de publicidad de las normas y seguridad jurídica, deben ser objeto de publicación oficial. Por consiguiente, ha sido seleccionado por la oficialidad de la fuente.

El periódico diario HOY (http://www.hoy.es/es), por su tradición y características. Fundado en 1933 con tres características bien definidas que ha sabido conservar a lo largo de los años: ser extremeño y para los extremeños, tener cobertura y difusión regional y mantenerse en vanguardia de la tecnología.

El Periódico Extremadura (http://www.elperiodicoextremadura.com/), por su tradición y espíritu. Manteniéndose fiel a sus lectores, ha sido siempre un reflejo de la actualidad y la historia de Extremadura. Sus más de 90 años de vida, lo convierten en el diario decano de la Región. Durante todos estos años, los avances tecnológicos han sido muchos, pero, según señala el propio medio, el espíritu sigue siendo el mismo que se implantó desde el primer día: un trabajo periodístico serio, recto, riguroso y siempre encaminado al servicio de la sociedad extremeña

A continuación, se decide el periodo de análisis de las noticias. Teniendo en cuenta el objetivo de esta investigación, se seleccionan cuatro años divididos en dos periodos: 2007 y 2008; 2012 y 2013. Se realizan las consultas sobre bibliotecas rurales en los tres medios de comunicación on line ${ }^{4}$ y en los cuatro años de análisis durante los meses de

4Interfaz de consulta: DOE http://doe.juntaex.es/busquedas/bus_avanzada.php, HOY http://www.hoy.es/hemeroteca/, El Periódico de Extremadura http://www.elperiodicoextremadura.com/noticias/hemeroteca.php. 
octubre, noviembre y diciembre de 2013, y se obtiene un total de 1196 referencias sobre bibliotecas rurales cuyo contenido es leído minuciosamente para comprobar su exactitud con la temática investigada ya que, a veces, el titular o los primeros párrafos no expresan exactamente el contenido de la noticia. Tras este filtro, se selecciona un conjunto final de 300 noticias sobre bibliotecas rurales lo que denota un $25 \%$ de coherencia entre las noticias publicadas y su temática real. Para el tratamiento de las noticias se diseña una base de datos con la que se trabaja conformada por los siguientes campos: Id (número de la noticia); Fecha (data de publicación); Origen (medio de publicación: DOE, HOY, El Periódico de Extremadura); Ámbito geográfico (nacional, regional, provincial); Tipo de biblioteca (rural); Fecha de la consulta; Enlace a la noticia (URL); Titular (título); Noticia (primeros párrafos) y Comentario (del analista).

\section{Resultados y Discusión}

Seguidamente, se muestran los resultados de las 300 noticias sobre bibliotecas rurales de forma integrada, combinando las variables estudiadas con el año de publicación: noticias por tipo de biblioteca/año, noticias por ámbito de la noticia/año, noticias por medio de publicación/año.

\section{Tipo de biblioteca/año de publicación}

En primer lugar, la tabla 1 y su figura 1 correspondiente (porcentual) muestra el número de noticias halladas por tipo de biblioteca en cuestión (rural) en cada año de análisis. En la figura se advierte que las bibliotecas rurales alcanzan su mayor porcentaje de noticias en el año 2007 (35\% bibliotecas rurales). Se podría pensar que en el año 2007 la crisis económica del país aún se encontraba en estado embrionario, por lo que las bibliotecas no se había hecho eco de la misma. Se observa un descenso acusado de noticias en el año 2012 respecto a los años anteriores. Es muy probable que la importante reducción de los presupuestos por la crisis, unido a la presencia monotemática en los medios de comunicación de noticias relativas a la marcha de la economía, influyese sobre la reducción de las noticias relativas a las bibliotecas rurales en los últimos años. 
Tabla 1. Noticias por tipo de biblioteca/año de publicación

\begin{tabular}{|l|r|r|r|r|r|}
\hline $\begin{array}{c}\text { Tipo de } \\
\text { biblioteca }\end{array}$ & $\mathbf{2 0 0 7}$ & $\mathbf{2 0 0 8}$ & $\mathbf{2 0 1 2}$ & $\mathbf{2 0 1 3}$ & Total \\
\hline B. Rurales & 105 & 95 & 33 & 67 & 300 \\
\hline
\end{tabular}

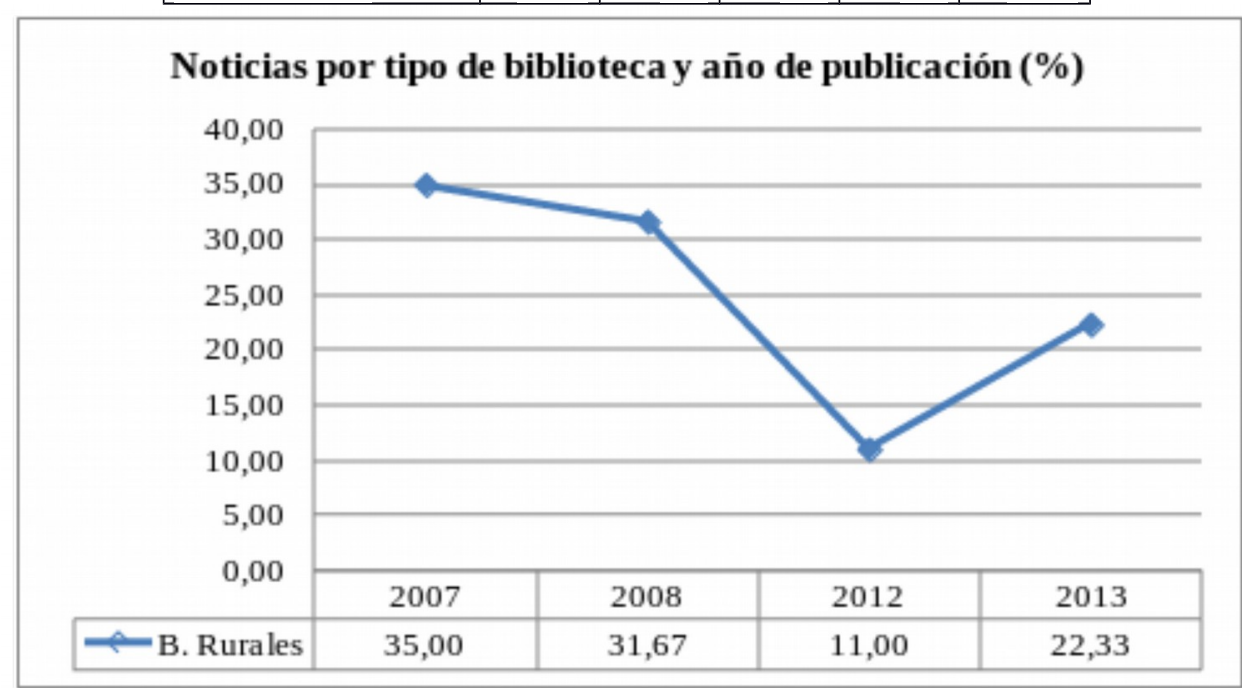

Figura 1. Porcentaje de noticias por tipo de biblioteca/año de publicación.

Para ver con mayor exactitud la evolución de las noticias se calcula la Tasa de Variación (TV) sobre los porcentajes de la figura 1. Las noticias sobre bibliotecas rurales tienen una TV negativa en 2008 respecto a 2007 de $-9,52 \%$ y en 2013 alcanzan una tasa positiva del $103 \%$ respecto a 2012.

\section{Ámbito de la noticia/año de publicación}

Si se disgregan las 300 noticias sobre bibliotecas rurales según su ámbito (tabla 2 y representación gráfica porcentual en figura 2), se observa que en todos los años, el mayor número de noticias sobre bibliotecas rurales hace referencia a la provincia de Badajoz (con mayor densidad de población), seguido de Cáceres. Es normal que estas dos provincias promuevan un mayor número de noticias sobre bibliotecas rurales (enfocadas, sobre todo, al fomento de la lectura y otras actividades de dinamización) que las de ámbito nacional o regional. Por ello, a gran distancia se encuentra el ámbito regional y, por último, el nacional que no recibe ninguna noticia. 
Tabla 2. Noticias sobre bibliotecas rurales por ámbito/año

\begin{tabular}{|l|r|r|r|r|r|}
\hline \multicolumn{1}{|c|}{$\begin{array}{c}\text { Ámbito de la } \\
\text { Noticia }\end{array}$} & $\mathbf{2 0 0}$ & $\mathbf{2 0 0}$ & $\mathbf{2 0 1}$ & $\mathbf{2 0 1}$ & \multicolumn{1}{c|}{$\mathbf{2}$} \\
\hline Nacional & 0 & 0 & 0 & 0 & Total \\
\hline Regional & 4 & 6 & 3 & 1 & 14 \\
\hline Cáceres & 38 & 36 & 8 & 26 & 108 \\
\hline Badajoz & 63 & 53 & 22 & 40 & 178 \\
\hline Total & 105 & 95 & 33 & 67 & 300 \\
\hline
\end{tabular}

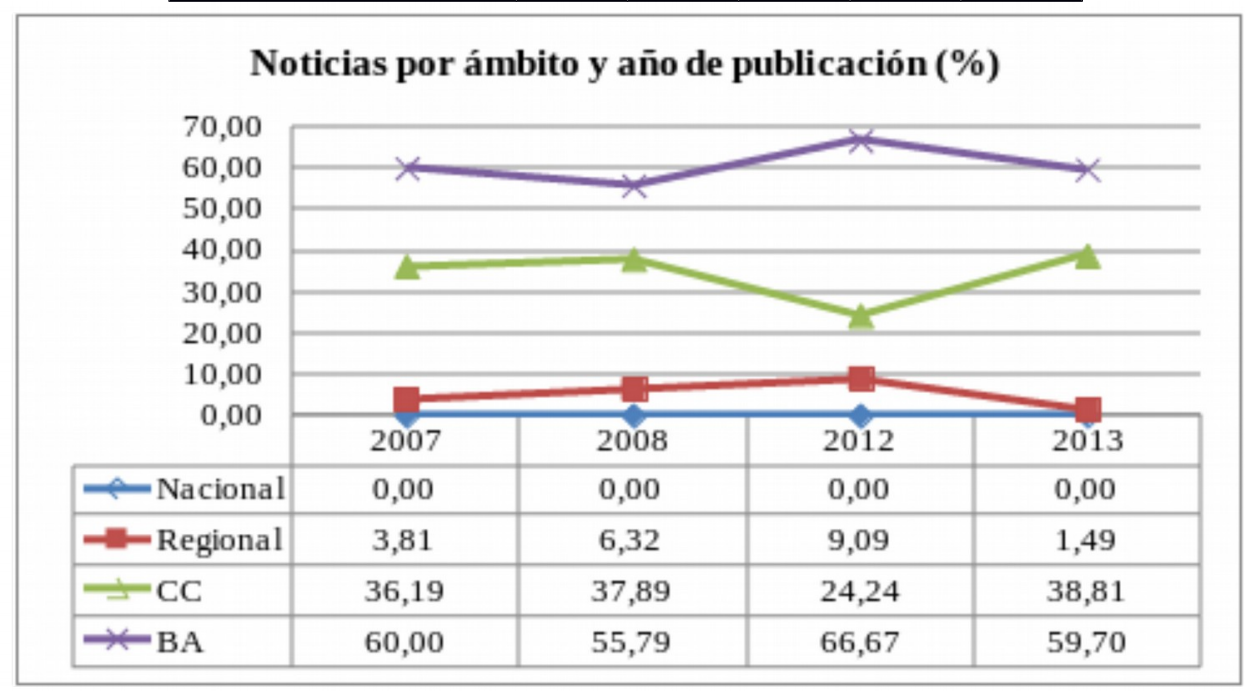

Figura 2. Porcentaje de noticias por ámbito/año de publicación

\section{Medio de publicación/año de publicación}

En la tabla 3 y su representación gráfica porcentual correspondiente (figura 3) se observan las noticias sobre bibliotecas rurales según su medio de publicación. En todos los años, el mayor número de noticias sobre bibliotecas rurales se publican en el diario $H O Y$, a gran distancia, en El Periódico Extremadura y, en último lugar, en el DOE (que no publica ninguna noticia ni en 2007 ni en 2013). 
Tabla 3. Noticias sobre bibliotecas rurales por medio de publicación/año

\begin{tabular}{|c|c|c|c|c|c|}
\hline $\begin{array}{l}\text { Medio de } \\
\text { Publicación }\end{array}$ & $\begin{array}{l}200 \\
7\end{array}$ & $\begin{array}{c}200 \\
8\end{array}$ & $\begin{array}{c}201 \\
2\end{array}$ & $\begin{array}{c}201 \\
3\end{array}$ & Total \\
\hline DOE & 0 & 4 & 2 & 0 & 6 \\
\hline HOY & 99 & 83 & 25 & 52 & 259 \\
\hline $\begin{array}{l}\text { Periódico } \\
\text { Extremadura }\end{array}$ & 6 & 8 & 6 & 15 & 5 \\
\hline Total & 105 & 95 & 33 & 67 & 300 \\
\hline
\end{tabular}

Noticias por medio de publicación y año de publicación (\%)

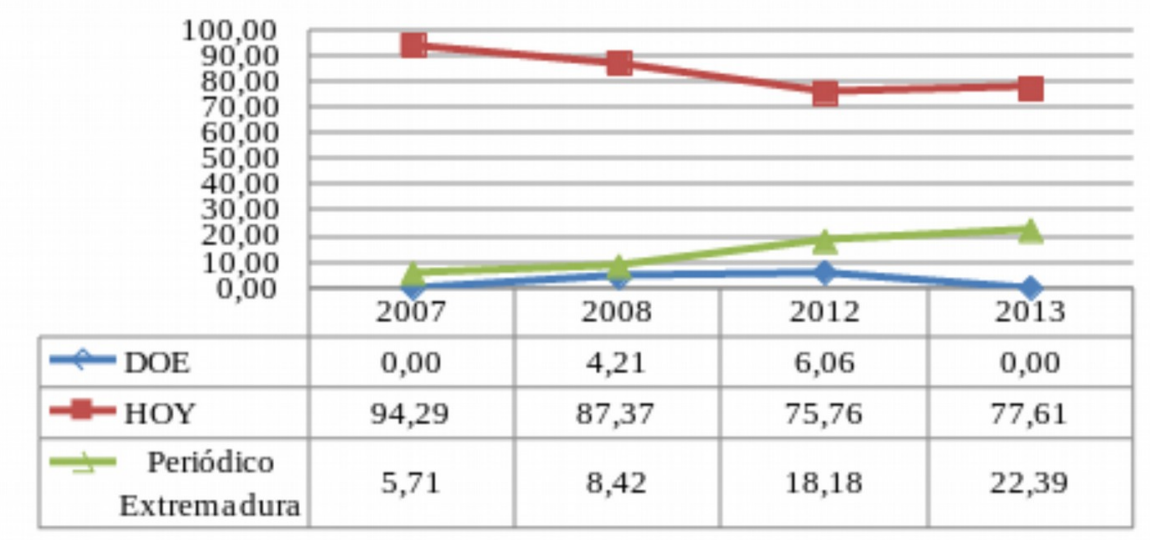

Figura 3. Porcentaje de noticias por medio/año de publicación

\section{Conclusiones}

Las bibliotecas rurales constituyen un pilar del patrimonio cultural colectivo de las regiones predominantemente rurales, como es el caso de la Comunidad Autónoma de Extremadura. Sin embargo, existe una laguna considerable en los estudios específicos sobre este tipo de bibliotecas. Por ello, en este trabajo se aportado una investigación sobre las mismas analizado la evolución y el impacto que dichas bibliotecas tienen en la sociedad extremeña a través de las noticias publicadas sobre ellas en diversos medios de comunicación muy representativos de la Región.

En muchos medios de comunicación, para tener la certeza de trabajar con noticias realmente referidas a la temática que indica su titular o sus primeros párrafos, es preciso profundizar en la noticia. En la presente investigación, solo el $25 \%$ de las noticias recuperadas en las consultas sobre bibliotecas rurales en los años 2007 y 2008; 2012 y 
2013, resultaron pertinentes para el tema de investigación, lo que refleja la necesidad de analizar exhaustivamente las noticias.

El mayor número de noticias sobre bibliotecas rurales se produce en el año 2007 (35\%), a partir de entonces se observa una caída en 2008 (31,67\%), muy acentuada en 2012 (11\%). Los datos de 2013 ofrecen una inversión de la tendencia que, si bien llega a representar el 22,33\% del total de las noticias, queda lejos del peso específico de las mismas antes de la crisis económica del país. Es decir, la evolución sufrida marca una tendencia general descendente en el tiempo, con un repunte en 2013. Es muy probable que la importante reducción de los presupuestos por la crisis económica, unido a la presencia monotemática en los medios de comunicación de noticias relativas a la marcha de la economía, influyese sobre la reducción de las noticias relativas a las bibliotecas en los últimos años. Sin embargo, tras calcular la Tasa de Variación en los dos periodos de análisis independientemente, se observa una diferencia de tendencia que refleja la propia situación económica de España durante estos últimos años, ya que, se obtiene una Tasa de Variación negativa del $-9,52 \%$ en 2008 respecto a 2007, pero una tasa positiva del 103\% de 2013 respecto a 2012.

Las noticias sobre bibliotecas de ámbito provincial obtienen el mayor porcentaje (Badajoz 59,33\% y Cáceres 36\%), seguidas de las regionales (4,67\%). Sin duda, estos resultados están condicionados por las fuentes: medios regionales donde el alcance principal de las noticas es, con mucho, regional. Las noticias provinciales se centran en las bibliotecas rurales, sobre todo, en aspectos relacionados con la animación a la lectura y extensión cultural en estos centros.

El diario $\mathrm{HOY}(86,33 \%)$ es el medio que acumula el mayor porcentaje de noticias sobre bibliotecas rurales consultadas seguido, a gran distancia, de El Periódico Extremadura $(11,67 \%)$ y, por último, del Diario Oficial de Extremadura (DOE) (2\%), donde aparecen, sobre todo, referencias sobre dotación económica para el fomento a la lectura.

Con el fin de dar visibilidad a estas bibliotecas que juegan un papel tan importante en el medio rural, sería recomendable ampliar el estudio abarcando otros años de análisis y 
otras zonas de la geografía española. Además, la metodología presentada en este trabajo también sería extrapolable a otros ámbitos extranjeros.

\section{BIBLIOGRAFÍA}

Boza Puerta, M., y Sánchez Herrador, M. A. (2004). Las bibliotecas en las Misiones Pedagógicas. Boletín de la Asociación Andaluza de bibliotecarios,74 (Marzo), 41-51.

Busto Fidalgo, M. (2009). Biblioteca rural y desarrollo sostenible. El caso de la Biblioteca Pública Municipal Menéndez Pelayo de Castropol (Asturias). En: III Encuentro de Bibliotecas y Municipio: Los servicios bibliotecarios en zonas rurales (pp. 67-77). Madrid: Ministerio de Cultura.

Busutil Rivera, G. (2011). Bibliotecas rurales. Mercurio: panorama de libros, 128: 17.

Cano Vers, M. (2009). El servicio de lectura pública. Los pequeños municipios de la provincia de Barcelona. En: III Encuentro de Bibliotecas y Municipio: Los servicios bibliotecarios en zonas rurales (pp. 175-185). Madrid: Ministerio de Cultura.

Cid Ruiz, J. C. y Fraile de la Hoz, M. (2009). La Biblioteca Municipal de Chapinería. Espacio de ocio, comunicación e información. En: III Encuentro de Bibliotecas y Municipio: Los servicios bibliotecarios en zonas rurales (pp. 79-103). Madrid: Ministerio de Cultura.

Comalat Navarra, M., y Reyes Camps, L. (2001). Los servicios bibliotecarios en zonas rurales: una propuesta de actuación viable ( $4^{\mathrm{a}}$ ed.). Barcelona: Diputación.

Ford, B.J. (2002). Todos son bienvenidos: la biblioteca pública como espacio de integración ciudadana. En I Congreso Nacional de Bibliotecas Públicas: La biblioteca pública, portal de la sociedad de la información (pp. 191-200). Madrid: Ministerio de Cultura.

García Gómez, F. J. (2004). La biblioteca pública española en el ámbito rural: una solución para problemas de exclusión. Boletín de la $A N A B A D, 54$ (1-2), 115-132. Disponible en 
http://www.anabad.org/archivo/docdow.php?id=145 [Consultado: 9 de diciembre de 2014].

García Gómez, F.J., y Díaz Grau, A. (2001). La biblioteca pública como instrumento para el desarrollo local y social de las poblaciones rurales. Boletín de la $A N A B A D, 51$ (1): 143162.

Germanaud, M.C. y Rappaport, G. (1988.) Crear y animar una biblioteca: en el medio rural, en las pequeñas poblaciones, en el hospital y en la empresa. Madrid: Pirámide.

Gómez Gómez, M.A., Gómez Hernández, J.A. y Lluch Baixauli, L. (1997). La imagen de las Bibliotecas Municipales en la prensa Regional de Murcia. En: VI Congreso Nacional de ANABAD: Archivos, Bibliotecas, Centros de Documentación y Museos en el Estado de las Autonomías (pp. 441-450). Murcia: ANABAD; Consejería de Cultura y Educación.

Gómez Santamaría, J.A. (2009). Aula telemática en la Biblioteca Municipal de Elciego. La biblioteca, centro de información, formación y autoaprendizaje. En: III Encuentro de Bibliotecas y Municipio: Los servicios bibliotecarios en zonas rurales (pp. 23-30). Madrid: Ministerio de Cultura.

González-Mateos, I. y Faba-Pérez, C. (2013). Globalización y acceso a la información en las bibliotecas escolares de Extremadura. En III VI Encuentro Ibérico EDICIC 2013 (pp. 1159-1169). Oporto: Faculdade de Letras.

Haines, R. y Calvert, P.J. (2009). Is isolation a problem? Issues faced by rural libraries and rural library staff South Australia. Australian Library Journal, 8 (4). Disponible en: http://eric.ed.gov/?id=EJ939088. [Consultado: 25 de febrero de 2014].

Hidalgo Mateos, A., Pérez Pulido, M.., Cebrián Solís, R. y Gordo Sánchez, A.S. (2012). Bibliotecas Rurales en Extremadura: análisis del valor y de su grado de implantación. Cáceres: Fundación Caja de Extremadura; Universidad de Extremadura. 
Hoffman-Pfeffer, C. (1987). Information needs of rural populations: implications for planning library/information services. Library Review, 36(2): 95-109.

Llano Caelles, X. (1997). La biblioteca en el mundo rural: reflexiones. Gijón: Trea

Lorenzo Jiménez, J. (2009). Castilla-La Mancha: soluciones para el servicio bibliotecario en el medio rural. En III Encuentro de Bibliotecas y Municipio: Los servicios bibliotecarios en zonas rurales (pp. 161-174). Madrid: Ministerio de Cultura.

Manifiesto de la IFLA/UNESCO sobre Internet. (2006). Disponible en http://archive.ifla.org/Faife/policy/iflastat/Internet-ManifestoGuidelines-es.pdf. [Consultado: 17 de enero de 2014].

Mehra, B., Black, K. y Shu-Yueh, L. (2010). Perspectives of East Tennessee's Rural Public Librarians about the Extent of Need for Professional Library Education: A Pilot Study. Journal of Education for Library and Information Scienc, e 51(3): 142-158.

Moliner Ruiz, M. (2010). La organización de una red de bibliotecas en la región de Valencia: fragmento. Educación y biblioteca, 22(175): 46-48.

Yuan, Y. (2011). Libraries on the way. Beijing Review, 54(48): 26-27.

Wigell-Ryynänen, B. (2009). La biblioteca como centro de servicios múltiples para zonas rurales. En III Encuentro de Bibliotecas y Municipio: Los servicios bibliotecarios en zonas rurales (pp. 13-20). Madrid: Ministerio de Cultura. 